\title{
A Glimpse in the Future of Malignant Mesothelioma Treatment
}

\author{
Gaetano Pezzicoli ${ }^{1}$,Mimma Rizzo ${ }^{2}$, Martina Perrone ${ }^{1}$, Silvia Minei ${ }^{1}$, Luciano Mutti ${ }^{3+}$ and \\ Camillo Porta ${ }^{1,2 * t}$
}

${ }^{1}$ Department of Biomedical Sciences and Human Oncology, University of Bari Aldo Moro, Bari, Italy, ${ }^{2}$ A.O.U. Consorziale Policlinico di Bari, Bari, Italy, ${ }^{3}$ Italian Group for Research and Therapy for Mesothelioma (GIMe), Voghera, Italy

Malignant mesothelioma (MMe) is a rare neoplasm with few therapeutic options available. The landscape of effective therapy for this disease remained unchanged in the last two decades. Recently, however, the introduction of Immune Checkpoint Inhibitors (ICls) led to small, but nevertheless, promising improvements. However, many efforts are still needed to radically improve the prognosis of MMe. In this review, we analyze all those therapeutic strategies for MMe that are still in a preclinical or early clinical phase of

OPEN ACCESS

Edited by:

Steven Eugene Mutsaers, University of Western Australia, Australia

Reviewed by: Maricla Galetti, National Institute for Insurance Against Accidents at Work (INAIL), Italy Elisa Giovannetti, VU Medical Center, Netherlands

*Correspondence: Camillo Porta camillo.porta@gmail.com orcid.org/0000-0003-2412-1563

tThese authors share senior authorship

Specialty section:

This article was submitted to Pharmacology of Anti-Cancer Drugs, a section of the journal

Frontiers in Pharmacology

Received: 04 November 2021 Accepted: 02 December 2021

Published: 15 December 2021

Citation:

Pezzicoli G, Rizzo M, Perrone M, Minei S, Mutti L and Porta C (2021) A Glimpse in the Future of Malignant Mesothelioma Treatment.

Front. Pharmacol. 12:809337. doi: 10.3389/fphar.2021.809337 development. In particular, we focus on novel antiangiogenic drugs and their possible combination with immunotherapy. Furthermore, we describe also more complex strategies such as microRNA-loaded vectors, oncolytic viruses, and engineered lymphocytes.

Keywords: mesothelioma, immune checkpoint inhibitors, antiangiogenics, mesothelin, microRNA, oncolytic viruses, CAR-T cells

\section{INTRODUCTION}

Malignant Mesothelioma ( $\mathrm{MMe}$ ) is commonly considered a rare cancer. However, it is estimated to affect 40.000 people worldwide, with its incidence growing decade after decade (Odgerel et al., 2017; Keshava et al., 2019). MMe onset is heavily correlated with professional exposure to asbestos, and its low survival rate makes it the deadliest occupational disease ever known. A decrease in the number of new diagnoses in western industrialized countries is expected in the next few decades, due to governments programs of asbestos banishment. On the other hand, a dramatic rise is foreshadowed in all those second and third-world countries in which no action against this cancerogenic mineral has been taken yet.

MMe first clinical manifestation is often dyspnea due to pleural effusion. Less common presentations include chest pain or systemic symptoms such as fever, weight loss, or night sweats. The onset of these signs in a patient with a known history of professional exposure to asbestos must be seen as a red flag. In this setting, a chest computed tomography (CT) scan should be performed. Whenever pleural effusion and pleural nodules are detected through imaging, the next step consists of surgical biopsy or CT-guided biopsy. Even if the cytologic diagnosis can be very effective and less invasive, tissue biopsy is still to be preferred since it allows the detection of peculiar architectural patterns that have a prognostic role. Histological workup will differentiate mainly between three forms of MMe: epithelioid, sarcomatoid, and biphasic, with sarcomatoid differentiation being a negative prognostic index (Nicholson et al., 2020).

Even if metastases are a rare phenomenon in $\mathrm{MMe}$, this tumor has a very poor prognosis due to the respiratory function impairment that it causes. Very few chemotherapy agents have shown some efficacy against this disease, and no major improvements in its treatment occurred in the last 
two decades. Recently, however, something new emerged, leading to the introduction of a whole new class of drugs in the management of MMe: the immune checkpoint inhibitors (ICI).

\section{CURRENT AND UPCOMING STANDARD OF CARE}

\section{Surgery}

Pleural decortication and extrapleural pneumonectomy are the two major surgical techniques involved in the treatment of MMe. Unfortunately, no consensus exists about the role of surgery in the management of $\mathrm{MMe}$, since there is a lack of prospective studies. The MARS trial is one of the few studies of this type and it concludes that there is no significant survival advantage in patients undergoing extrapleural pneumonectomy, followed by palliative chemotherapy, compared with patients receiving palliative chemotherapy alone $(\mathrm{HR}=1.90,95 \% \mathrm{CI}=$ 0.92-3.93; $p=0.082$ ) (Treasure et al., 2011). The precise role of pleural decortication is being investigated within the MARS-2 trial, which is still recruiting and its completion is estimated in late 2022 (NCT02040272).

What emerges from retrospective analyses is the importance of patient selection. Factors such as age, comorbidities, and performance status are important guidance in this choice. Also, histology plays an important role: many experts point out that sarcomatoid $\mathrm{MMe}$ should never receive surgical treatment, while the debate is still open for biphasic MMe (Ricciardi et al., 2018).

Neoadjuvant treatments have been the object of active investigation, yielding mixed results. As a whole, neoadjuvant chemotherapy granted no survival advantage (Voigt et al., 2020). Neoadjuvant radiotherapy was recently evaluated in a phase II trial (SMART), that showed only the feasibility of this technique (Cho et al., 2021).

However, even though there is a certain interest in the surgery of MMe, it should be noted that the vast majority of patients are be suitable for surgical intervention, and will receive a palliative systemic therapy only.

\section{Palliative Chemotherapy}

Patients unsuitable for surgery, usually receive first-line chemotherapy. The combination of Pemetrexed and Cisplatin represents the backbone of the treatment in this setting since a phase III trial demonstrated a progression-free survival (PFS) improvement for the combination versus cisplatin alone (5.7 vs. 3.9 months, $p=0.001$ ), back in 2003 (Vogelzang et al., 2003). Carboplatin can be used in patients unfit for Cisplatin (Ceresoli et al., 2006). There is currently no evidence supporting maintenance therapy for patients achieving disease control, with many studies demonstrating no benefit from the prosecution of Pemetrexed, the latest being the CALGB 30901 trial (Dudek et al., 2020). The addition of antiangiogenics to chemotherapy has been considered since MMe is well known for its high VEGFR expression (Aoe et al., 2006). The MAPS trial observed a slight advantage in overall survival (OS) for patients receiving Pemetrexed, Cisplatin, and Bevacizumab compared with patients receiving chemotherapy alone (18.8 vs. 16.1 months, HR 0.77, $p=0.0167)$. However, this improvement comes at the cost of a higher rate of grade 3-4 adverse events (Zalcman et al., 2016). As a whole, Bevacizumab is not FDA approved in this setting.

Only a few options exist in the common clinical practice for patients with progressive disease after first-line therapy. For patients experiencing progression more than 6 months from the first-line treatment, rechallenge with pemetrexed and a platinum compound is advised. Bearz et al. demonstrated that in this setting, the rechallenge strategy allows a disease control rate (DCR) of $66 \%$, with a PFS of 5.1 months and an OS of 13.6 months (Bearz et al., 2012). However, patients suitable for rechallenge represent a minority. For patients experiencing early progression, monotherapy with Gemcitabine or Vinorelbine seems to be one of the few strategies offering a minimal survival improvement (Zucali et al., 2014). Recently, the RAMES trial showed a possible role for antiangiogenic drugs in this setting. The combination of Gemcitabine and Ramucirumab (an anti-VEGR2 monoclonal antibody) was compared with Gemcitabine alone in patients beyond the first line of treatment, obtaining a significant OS improvement (13.8 vs. 7.5 months, HR $0.71,70 \%$ CI $0.59-0.85 ; p=0.028$ ) (Pinto et al., 2021), with an acceptable safety profile. However, this study has been criticized especially for the positive selection of the patients enrolled (Porta et al., 2021).

\section{Immune Checkpoint Inhibitors}

The introduction of ICIs can be considered a turning point in the management of MMe. In fact, after many studies showing a potential role for this treatment in the second and third lines, (Fennell et al., 2018; Popat et al., 2020) the Checkmate-743 trial demonstrated an important clinical improvement with an ICI in the first line setting (Baas et al., 2021). Specifically, this study randomized 605 patients with advanced MMe to receive either Nivolumab (an anti-PD-L1 monoclonal antibody) plus Ipilimumab (an anti-CTLA4 monoclonal antibody), or platinum-based chemotherapy. The OS was 18.1 vs. 14.1 months (HR 0.74, $p=0.002$ ) and the frequency of the adverse events was comparable between the two groups. Subgroup analyses showed benefits for the immune combo in nearly every considered subgroup, with only a minor (but nevertheless significant) improvement for patients with PD-L1 negative tumors, as well as in elderly patients. A finding of high interest is the heightened efficacy of the immune combo compared with chemotherapy in patients with non-epithelioid histologies ( $\mathrm{OS}=18.1$ vs. 8.8 months, $\mathrm{HR}=0.46$ ). On the other hand, it should be noted that this trial has some limitations, since it did not enroll patients with an ECOG Performance Status of 2, which are not a rare among the population of MMe patients undergoing active treatments. In the meantime, the study population was still so fragile that small changes in the randomization could have lead to different statistical conclusions.

Other trials are trying to expand the role of ICIs in MMe. The DREAM phase II trial showed a 6.7 months PFS and a 20 months OS in naïve MMe patients treated with Durvalumab (an anti-PDL1 monoclonal antibody) plus Pemetrexed and Cisplatin (Nowak 
TABLE 1 | Clinical trials involving novel therapeutic strategies for MPM.

\begin{tabular}{|c|c|c|}
\hline Strategy exploited & Study population & Study design \\
\hline
\end{tabular}

Novel cytotoxic agents

Arginine deiminase

30 patients with MPM, that had progression after a first-line platinumbased therapy

42 patients with MPM, that had progression after first-line platinumbased therapy or immunotherapy 386 treatment-naiive patients with MPM (ongoing enrollment)

Tyrosin-Kinase inhibitors

Antiangiogenics

Combination of $\mathrm{ICl}$ and antiangiogenics

DNA reparation impairment

microRNA

HDAC inhibitors

Novel

immunotherapies

24 patients with MPM, that had progression after first or second-line chemotherapy

62 patients with pretreated advanced neoplasms (including a cohort of 29 MPM)

54 patients with MPM, that had progression after a first-line platinumbased therapy

51 patients with MPM, that had progression after a first-line platinumbased therapy

92 treatment-naïve patients with MPM

18 patients with solid neoplasms, including MPM, that had progression after first-line therapy (ongoing enrollment)

400 treatment-naïve patients with MPM (ongoing enrollment)

20 patients with malignant peritoneal mesothelioma, that had progression after first-line platinum-based therapy 74 patients with MPM, that had progression after first-line platinumbased therapy

26 patients with MPM, that had progression after first-line platinumbased therapy

661 patients with MPM, that had progression after first or second-line chemotherapy

148 patients with pretreated

advanced neoplasms (including MPM)
248 patients with MPM, that had progression after first-line platinumbased therapy

35 treatment-naïve patients with MPM

27 patients with MPM, that had progression after first-line platinumbased therapy

Oncolytic viruses
13 patients with MPM, who received a maximum of one line of treatment
A phase II trial investigating the efficacy of BNC105P (tubulin-targeting agent)

SAKK 16/17: phase II trial investigating the efficacy of Lubrinectidin (DNA

transcription inhibitor)

ATOMIC-meso: phase II/III trial investigating the efficacy of ADI-PEG20 (pegylated arginine deiminase) in combination with pemetrexed and cisplatin

A phase II trial investigating the efficacy of AZD4547 (FGFR1-3 inhibitor)

A phase I pharmacokinetic and pharmacodynamic study of GSK2256098 (focal adhesion kinase inhibitor) SWOG S0509: a phase II trial investigating the efficacy of Cediranib (pan-VEGFR inhibitor)

A phase II trial investigating the efficacy of

Cediranib (pan-VEGFR inhibitor)

SWOG S0905: a phase II comparison of Cediranib (pan-VEGFR inhibitor) + pemetrexed and cisplatin vs pemetrexed and cisplatin

PEMBIB: a phase I trial investigating safety and activity of Pembrolizumab and Nindetanib

BEAT-meso: a phase III trial of

Pemetrexed-Carboplatin-

Bevacizumab-Atezolizumab vs

Pemetrexed-Carboplatin-Bevacizumab

A phase II trial investigating the efficacy of Atezolizumab plus Bevacizumab

A phase II trial investigating the efficacy of Tamezostat (EZH2 inhibitor)

A phase I trial investigating safety and activity of TargomiRs (miR16-loaded non-living minicells)

VANTAGE-014: a phase III trial of

Vorinostat vs placebo

A phase I Dose-Escalation trial of

Anetumab Ravtansine (Anti-Mesothelin Antibody-Drug Conjugate)

A phase II trial investigating the efficacy of

Anetumab Ravtansine (Anti-Mesothelin

Antibody-Drug Conjugate)

A phase I trial investigating safety and activity of CRS-207 (Listeria monocytogenes Expressing Mesothelin) with Chemotherapy

A phase I trial investigating safety and activity of intrapleural CAR-Tmeso plus

Pembrolizumab

A phase I/II trial investigating the efficacy of ORR 0\%, DCR $50 \%$ HSV1716 (oncolytic Herpesvirus)
ORR 3\%, DCR 43\%, mOS 8.2 months, Nowak et al. no grade $3-4 \mathrm{AE}$

(2013)

ORR $4 \%$, DCR $52 \%$, mOS

Metaxas et al. 11.1 months, mPFS 4.1 months

(2020)

Not yet published

NCT02709512

Discontinuation due to low 6-monthsPFS (12\%)

ORR 10\%, mPFS 3 months

Soria et al. (2016)

ORR 9\%, DCR 42\%, mOS 9.5 months, mPFS 2.6 months. $91 \%$ of patients required dose reduction

ORR 10\%, DCR 67\%, mOS

4.4 months, mPFS 1.9 months. $87 \%$ of patients reported grade $3 / 4 \mathrm{AE}$

Increased DCR (50 vs $20 \%$ ) and mPFS (7.2 vs. 5.6 months) in the cediranib arm. No differences in mOS. More AE in the ceridanib arm

Not yet published

NCT02856425

Garland et al. (2011)

Campbell et al.

(2012)

Tsao et al. (2019)

Not yet published

NCT03762018

ORR 40\%, 1-year-PFS 61\%, 1-yearOS $85 \%$

Raghav et al. (2021)

ORR 3\%, 12-weeks-DCR 51\%, 24 weeks-DCR 25\%

Marjorie et al. (2020)

ORR 5\%, DCR 73\%, mOS 6.8 months van Zandwijk et al. (2017)

No significant improvement in OS with Vorinostat (mOS 7.5 vs. 6.7 months)

ORR 8\%, DCR 52\%, best responses in tumor with high mesothelin expression

(2020)

Not yet published

NCT02610140

ORR 57\%, DCR 86\%, mOS

14.7 months, mPFS 7.5 months

Hassan et al. (2019)

mOS 23.1 months, 1-year-OS 83\%

Adusumili et al. (2021)

Danson et al. (2020)

Abbreviations: mOS, median overall survival; mPFS, median progression-free survival; ORR, objective response rate; DCR, disease control rate; $A E$, adverse event. Bold values are the experimental drug(s) evaluated in each study. 
et al., 2020). This could be a reasonable therapeutic option for patients with epithelioid histology. Finally, the phase III BEATmeso trial is currently enrolling naïve patients with $\mathrm{MMe}$ to evaluate the combination of Pemetrexed, Carboplatin, Atezolizumab, and Bevacizumab (NCT03762018). Results are expected in early 2024 .

As emerges from this brief overview, in the last few years many new therapeutic targets approached the common clinical management of MMe. After more than 15 years without significant changes, the standard of care has been ultimately modified by these novel findings. On the other hand, it should be considered that MMe still remains a disease with few overall therapeutic options, even including the most recent breakthroughs. Therefore, the research for new targets and, consequentially, new therapeutic strategies, will be an important aim for the next decade.

\section{NOVEL AGENTS AND COMBINATIONS}

The results of the studies analyzed in this section are summarized in Table 1.

\section{Novel Cytotoxic Chemotherapy Agents}

Despite the above recent improvements, the search for novel chemotherapeutic agents continues. Among the many compounds tested in $\mathrm{MMe}, \mathrm{BNC105 \textrm {P }}$ is surely one of the most interesting.

BNC105P is a tubulin targeting agent that disrupts the vascular architecture in solid tumors. It has been demonstrated to enhance the effect of VEGFR-inhibitors and mTOR-inhibitors in renal and breast cancer (Inglis et al., 2014). This drug was also tested in MMe, witin a phase II trial enrolling patients that had progressed on first-line platinum-based therapy (Nowak et al., 2013). In spite of the absence of grade 3-4 adverse events, the reported ORR was just 3\%, with a DCR of $43 \%$, and a median OS of 8.2 months. As a whole, it is clear that BNC105P is not an option as a monotherapy. However, its good safety profile and the solid rationale behind its use in MMe are solid points that could justify further development of this agent within combination with other agents.

Another interesting compound is Lubrinectedin, an agent that is similar to Trabectedin and that showed good efficacy in small cell lung cancer that progressed beyond the first line of platinumbased therapy (Trigo et al., 2020). Lubrinectedin acts by binding specific DNA sites thus impeding the access of transcriptional machinery and by inhibiting the function of tumor-associated macrophages (Santamaría Nuñez et al., 2016). A preliminary in vitro study showed a high sensitivity of MMe cell lines to Lubrinectidin, regardless of the BAP1 status and histological subtype (Anobile et al., 2021). Specifically, the inhibition in tumor growth was caused by the stop of the cell cycle in the S-phase with the activation of the DNA damage response and consequent apoptotic cell death. Given these premises, Lubrinectedin was tested in the SAKK 17/16 phase II trial (Metaxas et al., 2020). MMe patients with at least one therapeutic line failure were given Lubrinectidin in monotherapy. Interestingly, immunotherapy was included among the possible previous therapeutic lines, making this trial one of the few available trials providing evidence of activity after ICIs. A median PFS of 4.1 months and a median OS of 11.1 months were reported. The safety profile was acceptable, with only a minor part of patients experiencing neutropenia and fatigue. These results are of great clinical interest since Lubrinectidin is among the few drugs that reported clinical efficacy after ICIs in MMe. Moreover, the possibilities for combination therapies including Lubrinectidin in this setting are completely open.

\section{Arginine Deiminase}

Pegylated arginine deiminase is a novel therapeutic agent. In the Argininosuccinate synthetase 1 (ASS1)-deficient tumors, the enzyme Arginine Deiminase triggers an extracellular arginine depletion, inducing a pro-survival metabolic reprogramming that redirects glucose into the serine/folate pathway directing the carbons from glucose into pyrimidine biosynthesis, thus sensitizing cells to death by the pyrimidine antimetabolite, such as pemetrexed (Singh et al., 2019). The ASS1 deficit was identified in up to $75 \%$ of non-epithelioid MMe. Hence the rationale of the ATOMIC-meso phase II/III trial (NCT02709512) in which the ADI-PEG20 (pegylated recombinant Arginine Deiminase) is being tested in combination with Pemetrexed and Cisplatin, in naïve nonepithelioid MMe patients. In the phase II part of the study, patients are enrolled in an ASS1-agnostic fashion, with an option to restrict enrolment to ASS1-deficient in phase III. The control arm for phase III will be the platinum doublet. It is estimated that the study will be completed in late 2022 .

\section{Tyrosine-Kinase Inhibitors}

In the last two decades, TKIs changed the clinical management of a number of neoplasms, including kidney cancer and non-small cell lung cancer (NSCLC). However, the TKIs that worked for NSCLC showed little-to-no efficacy in MMe (Garland et al., 2007). Only recently, the expansion in the knowledge of MMe-specific drivers allowed the development of more promising TKIs.

Fibroblast Growth Factor Receptors (FGFR) 1-4 are often overexpressed in MMe, with FGFR3 and 4 being also predictors of shorter OS (Vlacic et al., 2019). A phase II clinical trial studied the efficacy of the FGFR 1-3 inhibitor AZD4547 in 24 advanced MMe patients that progressed after 1 or 2 lines of chemotherapy. However, the primary endpoint was not met, with a 6-months PFS of a mere $12 \%$, which lead to study discontinuation (Lam et al., 2020).

Another potential therapeutic target could be the Focal Adhesion Kinase (FAK), which is known to be amplified in $\mathrm{MMe}$ and pancreatic cancer. Kanteti et al. demonstrated growth inhibition caused by FAK-inhibitors in an in vitro model of MMe (Kanteti et al., 2018). Based on this preclinical rationale, Soria et al. explored the role of the FAK-inhibitor GSK2256098 in a cohort of 62 patients with different solid tumors, within a phase Ib trial (Soria et al., 2016). The subgroup of patients with MMe $(n=29)$ had a mPFS of 
12 weeks, and 3 of them experimented partial responses, a quite interesting finding taken into account that the patients enrolled were heavily pretreated. Moreover, a slightly better response was observed in MMe characterized with Merlin loss. The acceptable safety profile and the preliminary efficacy data, make FAK a target worth further experimentation.

Cyclin-dependent Kinases (CDK) are another interesting target. In the last decade, $\mathrm{CDK} 4 / 6$ inhibitors have been gamechangers in the treatment of metastatic breast cancer (Spring et al., 2019). Aliagas et al. observed that MMe patients with CDK4/6 overexpression experience a shorter survival. Therefore they tested the effects of the CDK 4/6 inhibitors Abemaciclib and Palbociclib on in vitro and in vivo preclinical models of MMe. Both the inhibitors showed the capability of blocking the cell cycle in the G1 phase and inducing senescence (Aliagas et al., 2021). Moreover, Abemaciclib was tested in a mouse model of MMe, achieving a significant inhibition of tumor growth, especially when combined with radiation therapy (Seiji et al., 2018). Another evidence supporting the use of CDK 4/6 inhibitors in $\mathrm{MMe}$ comes from an in silico analysis showing that the resistance to PD1-blockade in this tumor often comes from the deletion of CDKN2A (Jang et al., 2021). The authors of this analysis argued that this resistance mechanism can be overcome through the inhibition of CDK 4/6, and proceeded to demonstrate this hypothesis in an in vivo murine model. The combination of daily oral administration of CDK4/6 inhibitors (Abemaciclib or Palbociclib) and intraperitoneal anti-PD-1 treatment markedly suppressed tumor growth, compared with anti-PD-1 or CDK4/6 inhibitor alone. Therefore, there is a strong rationale for the use of CDK4/6 inhibitors, maybe in combination with ICIs. Presently, the currently recruiting MiST trial (NCT03654833) includes an arm of MMe patients selected by the p16/INK4A deficiency, who will receive Abemaciclib in monotherapy as a treatment. First results are expected in late 2021 .

\section{Antiangiogenics in Monotherapy and in Combination}

Antiangiogenic drugs may play an important role in the management of MMe. The pan-VEGFR inhibitor Cediranib has extensively been tested in this disease. Initially, it was tested as a second-line monotherapy treatment, to be used after progression to platinum-based chemotherapy. The SWOG S0509 phase II trial reported an mOS of 9.5 months, with an ORR of 9\% (Garland et al., 2011). Due to the low efficacy and the high rate of dose reductions ( $91 \%$ of all the patients), the study was discontinued. Similar results were described in a phase II trial by Campbell et al., who reported an even lower mOS (4.4 months) (Campbell et al., 2012). More recently, however, Cediranib was evaluated in combination with cisplatin and pemetrexed in chemotherapy-naïve $\mathrm{MMe}$ patients. The phase II SWOG S0905 compared the combination of cediranib with chemotherapy, yielding a PFS benefit in favor of the combination arm (7.2 vs. 5.6 months, HR 0.71, $p=0.062$ ), but no significant differences in OS (Tsao et al., 2019). As already seen in the RAMES trial, these studies seem to indicate a possible role for novel antiangiogenic therapies in $\mathrm{MMe}$, especially when combined with other cytotoxic drugs.

A different approach could be the combination of ICIs and antiangiogenics. As observed in kidney cancer, the effects of ICIs and antiangiogenic drugs are synergistic (Rini et al., 2019).

The PEMBIB trial (NCT02856425) is a phase Ib trial currently enrolling patients with different solid malignancies to receive a combination of Pembrolizumab and Nindetanib. The study includes a cohort of patients with $\mathrm{MMe}$ and it is currently reporting a favorable safety profile for the combination (Andreea et al., 2018). However, the completion is expected in 2026. Another study exploiting the combination of antiangiogenics and immunotherapy is the previously mentioned BEAT-meso trial, which is evaluating the combination of Atezolizumab, Bevacizumab, and chemotherapy. Neither this study will be completed soon. Recently, interesting evidence comes from a small study that demonstrated the activity of the combination of Atezolizumab plus Bevacizumab in a cohort of patients with peritoneal mesothelioma that had failed one previous platinum-based therapy line (Raghav et al., 2021). An ORR of $40 \%$ was reported, with a median duration of response of 12.8 months, a 1 -year-OS of $85 \%$, and a 1 -year-PFS of $65 \%$. The response seemed to be independent of the PD-L1 status and the principal resistance mechanism observed was the epithelial-tomesenchymal transition.

Whatsoever, more studies should be carried on in order to better understand the place for this combination in the future landscape of MMe therapies.

\section{Agents for MMe With Impairment in DNA Repair}

A high DNA instability has been documented in MMe (Ivanov et al., 2009). Specifically, asbestos exposure seems to correlate with a decreased activity of the DNA reparation system based on the Poli-ADP-Ribose Polymerase 1 (PARP1). As a counterproof, MMe cells often display a high grade of DNA damage, despite PARP1 being overexpressed (Tomasetti et al., 2011). It should hereby be highlighted that many MMe cases show the loss of the BRCA-associated protein 1 (BAP1), which is a deubiquitinase strictly implied in the BRCA-dependent DNA reparation system. Therefore MMe can be pictured as a tumor with a strong impairment in the DNA reparation. A preliminary in vitro study demonstrated that PARP inhibitors do not exert a strong cytotoxic effect on MMe cell lines, di per se (Rathkey et al., 2020). This can be explained considering that, as described before, PARP activity should already be minimal in MMe. However, the same study proved that in BAP1-deficient MMe, the combination of Temozolomide and PARP inhibitors can limit tumor growth. This may be due to the DNA damage caused by Temozolomide, which cannot be repaired by these tumor cells that lack the necessary enzymes. Given the analyzed rationale and the preclinical results, the clinical experimentation of this combination could theoretically lead to promising outcomes.

There is, however, another drug that could bring novelty in the management of BAP1-deficient MPM. Tazemetostat is a specific 
Oncolytic viruses

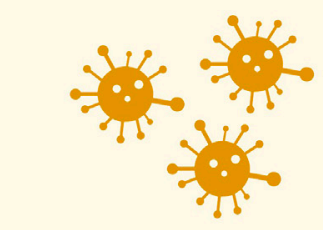

HSV17/16 is a replication restricted oncolytic herpes simplex virus

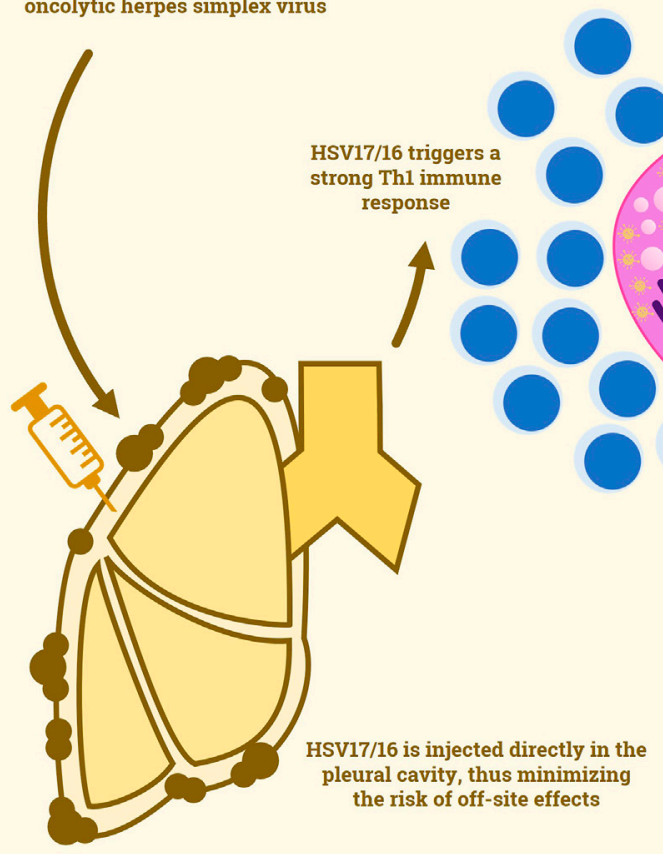

MMe cell

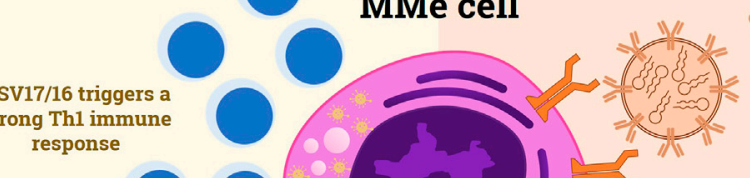

FIGURE 1 | A comparison between the mechanisms of action of oncolytic viruses and non-living minicells loaded with miRNA

inhibitor of the Enhancer of Zeste-Homolog 2 (EHZ2), a methyltransferase enzyme that plays a role in epigenetic regulation, by modulating the histones and by suppressing the transcription during cell division. The EHZ-203 phase II trial (NCT02860286) is evaluating the role of Tazemetostat in BAP1deficient $\mathrm{MMe}$, the rationale being the following: by inhibiting EHZ2, MMe will be unable to effectively suppress transcription, thus resulting in the production of aberrant proteins due to the DNA alteration linked to BAP1 loss (Marjorie et al., 2020). At the last update, the study reported a 12 -weeks DCR of $47 \%$, which is promising, considering that all the enrolled patients had failed at least one chemotherapy line. This trial both suggests a possible role for the combination of novel agents and presents an optimal paradigm for drug development in molecularly defined cohorts in mesothelioma.

\section{microRNA}

Since their discovery, microRNAs have always been considered a potential anti-tumoral target. These non-coding small RNA molecules usually play a role in the regulation of gene expression at a post-transcriptional level, by combining with specific proteins, thus creating ribozymes that suppress coding mRNAs (Peng and Croce, 2016). miRNAs were often found to be altered in many different cancer types. In MMe, some oncosuppressive role has been reported for miR-15/16. Reid et al. described a decreased expression of miR-15/16 in $\mathrm{MMe}$ tumor specimens and cell lines (Reid et al., 2013). Moreover, they demonstrated that restoring miR-16 function in MMe xenograftbearing mice leads to an inhibition of tumor growth correlated with the downregulation of $\mathrm{Bcl}-2$ and CCND1. The most important detail of this in vivo experimentation is the device used to restore the microRNA expression. In fact, Reid et al. engineered nonliving bacterial minicells to carry miR-16 and made them target MMe cells by positioning bispecific anti-EGFR antibodies on their surface (Figure 1). This system would later become known as TargomiRs. A phase I trial of TargomiRs in patients with pretreated MMe (NCT02369198) was recently published (van Zandwijk et al., 2017). The safety profile of this treatment resulted to be acceptable, with principal adverse events being lymphopenia, hypophosphatemia and increased transaminasemia. Despite an ORR of $5 \%$, the reported DCR was $73 \%$, with an mOS of 6.5 months. Therefore, TargomiRs 


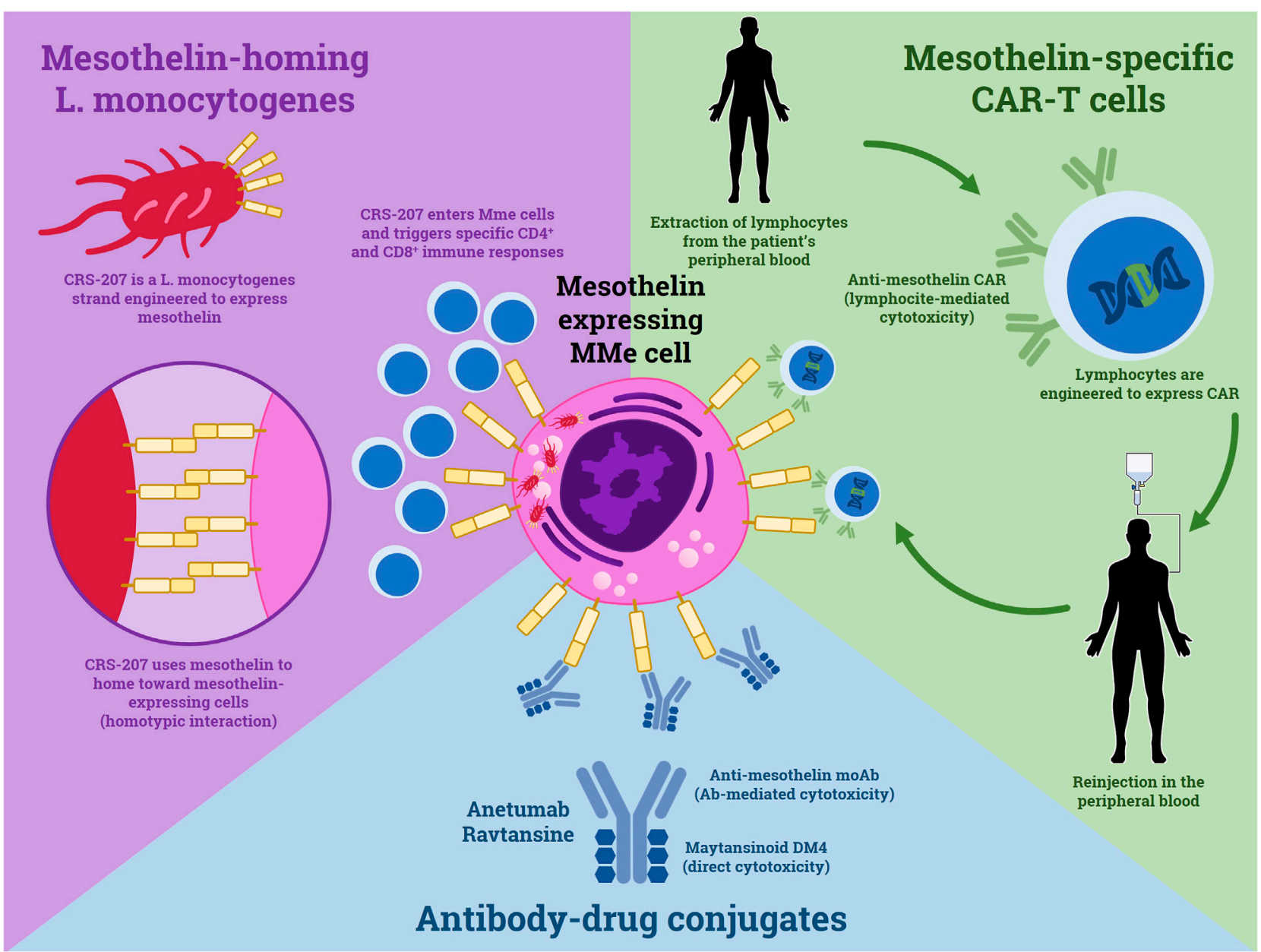

FIGURE 2 | Mechanisms of the novel immunotherapy strategies which use mesothelin as a target.

should be furtherly explored in the treatment of (NCT02369198), and combinations with immunotherapy or chemotherapy could be proposed.

\section{Histone Deacetylase Inhibitors}

HDAC inhibitors are still struggling to find their place in the clinical management of cancer. The in vitro studies show good tumor inhibition capabilities derived from their epigenetic manipulation. However, many clinical trials have been concluded without convincing evidence of their efficacy as a monotherapy. The scenario is not different for MMe. Panobinostat was demonstrated to inhibit mesothelioma cell growth both in vitro and in mice models (Crisanti et al., 2009). In this study, the same effect was observed in immunocompetent and immunodeficient mice, therefore it was assumed that it did not depend upon the immune system clearance mechanisms. These data sounded promising for a clinical application of HDAC inhibitors. In this context, the VANTAGE-014 study was promoted (Krug et al., 2015). In this phase III trial, MMe patients who experienced progression after a first-line platinum-based treatment were randomized to receive Vorinostat or placebo as a second-line treatment. The median OS did not significantly differ between the two treatments (30.7 vs. 27.1 weeks $p=0.86$ ). Even if this trial could be the tombstone on the use of HDAC inhibitors in MMe, a recent preclinical study rekindled the interest in this class of compounds. In an in vitro study, Bensaid et al. demonstrated that the combination of novel HDAC inhibitors and the hypomethylating agent Decitabine can induce the expression of specific immunogenic antigens on the surface of MMe cells and that these antigens are able to elicit specific immune responses (Bensaid et al., 2018). This effect, however, comes along with an increased PD-L1 expression. Therefore, this study foreshadows the possibility that a combination of HDAC inhibitors and ICIs could lead to increased efficacy of immunotherapy in MMe.

\section{Novel Immunotherapies}

The idea of using the immune system to target cancer has longly fascinated oncologists. In the last decade, the great success of ICIs demonstrated that this strategy could change the clinical practice for many different kinds of tumors. However, what has been done 
with ICIs is a mere scratch on the surface of what immunotherapy could produce. In fact, ICIs act by removing the natural boundaries of immune response thus allowing the immune system to overcome cancer immunoediting. However, more complex therapies that are currently under development, could take this concept to the next level. The strategies exploited in this chapter are summarized in Figure 2.

With respect to $\mathrm{MMe}$, the evolution of immunotherapy passes through the identification of a specific marker, possibly expressed on the surface of cancer cells. Mesothelin seems to fit this role. The function of this protein is not completely understood: it is well known its role as a heterotypic adhesion and its interaction with the CA-125 antigen (Bononi et al., 2015). Given its overexpression on MMe cells, mesothelin could be implied in contact-mediated survival. All these characteristics make it an ideal target.

The development of an anti-mesothelin antibody has longly been pursued. Many preclinical studies were carried on, with mixed results. However, very recently, the first anti-mesothelin antibody with a conjugated cytotoxic drug reached clinical experimentation. Anetumab Ravtansine is an anti-mesothelin antibody linked to an antimicrotubular agent (maytansinoid DM4) that was recently tested in a phase I dose-finding trial, demonstrating an acceptable safety profile (Hassan et al., 2020). The study included patients with many different mesothelinexpressing tumors, often heavily pretreated. Nevertheless, a disease control rate of $53 \%$ was reported. In particular, the MMe cohort was the one with the best results. The activity of this conjugate seems to be higher in those patients with a higher expression of mesothelin. Recently, a phase II trial was completed. In this study (NCT02610140), MMe patients who had progression after platinum-based chemotherapy were randomized to either Anetumab Ravtansine or Vinorelbine. However, the results are still undisclosed.

A less orthodox way of targeting mesothelin has been tried by Hassan et al. (2019). In a phase Ib trial, they utilized a nonvirulent strand of the bacteria Listeria monocytogenes engineered to express mesothelin, namely CRS-207. Exploiting the homotypic interaction of mesothelin molecules, CRS-207 reaches the MMe sites and here it triggers mesothelin-specific CD4+ and CD8+ T-cell responses, as demonstrated in preclinical studies (Le et al., 2015). In this phase Ib trial, patients with treatment-naïve MMe received induction with CRS-207, followed by up to 6 cycles of Cisplatin-Pemetrexed chemotherapy. Out of 35 patients, 31 had disease control, with 1 complete response and 19 partial responses. The median PFS and OS were 7.5 and 14.7 months, respectively. The most interesting part of this study is the comparison of pre and post CRS-207 biopsies, which showed a reduction of immunosuppressive phenomena and a switch of the immune infiltrate toward an active anti-cancer response. More studies on this novel strategy could feature combinations with other agents, leading to an even more effective response.

There is, however, an even more advanced strategy to target MPM cells that represents the pinnacle of next-level immunotherapies: the Chimeric Antigen Receptor $\mathrm{T}$ cells (CAR-T). Essentially, CAR-T are T lymphocytes engineered to express a chimeric receptor that is specific for a selected antigen. They represented a major breakthrough in the treatment of hematologic neoplasms in recent years. As for the management of MPM, some clinical trials featured mesothelin-specific CAR-T. In 2014, Beatty et al. exposed the first two cases of patients treated with CAR-Tmeso, a specific clone of CAR-T cells engineered to transiently express the CAR, thus limiting the possible onset of immune-related toxicities (Beatty et al., 2015). The report claims that the therapy is safe, feasible and it causes the onset of specific immune responses in the patient. CAR-Tmeso were successively tested in a phase I trial enrolling pretreated patients with mesothelin-expressing tumors, including $\mathrm{MMe}$, ovarian cancer, and pancreatic ductal adenocarcinoma (Haas et al., 2019). CAR-T cells were well tolerated and in the majority of patients, their DNA having been found in tumor biopsies. Moreover, this study demonstrated the importance of lymphodepletion before the infusion of CAR-T cells, since this strategy favored the expansion of the engineered lymphocytes. But it was in an even more recent study, that CAR-Tmeso showed their true efficacy. In the phase I trial by Adusumili et al., CAR-Tmeso were administered through intrapleural infusion in $25 \mathrm{MMe}$ patients that already received at least one line of therapy (Adusumilli et al., 2021). After these infusions, patients received immunotherapy with Pembrolizumab. The median OS from the first CAR-T infusion was 23.8 months, with $83 \%$ of patients alive at 1 year. Two patients exhibited a complete response at the PET scan. This study is of great interest not only for the management of MMe but also for the whole field of immunotherapy in solid tumors. The use of ICIs could represent the turning point in the struggle to export CAR-T therapy to nonliquid tumors. If these results will be confirmed by wider trials, the only limitations for this strategy will be its cost and its complexity.

\section{Oncolytic Viruses}

The use of viruses to attack tumor cells and trigger the host immune response has been purposed many times in the last 30 years, even if its realization proved to be difficult. Only recently, the first oncolytic viruses are reaching clinical practice. An example is T-VEC an oncolytic virus used in the treatment of advanced melanoma, that recently completed its phase III trial (Andtbacka et al., 2019).

One of the first reports of an oncolytic virus used against $\mathrm{MMe}$ is a 1997 preclinical study in which a replication restricted HSV-1 showed efficacy against in vitro $\mathrm{MMe}$ cell lines and it demonstrated to inhibit tumor growth in mice xenografts (Kucharczuk et al., 1997). More recently, other viruses were purposed for the same strategy. Li et al. reported the in vitro and in vivo efficacy of a measles virus engineered to carry the interferon-beta gene to enhance immune response and the sodium iodide symporter gene to track the virus diffusion through SPECT (Li et al., 2010). This virus was able to effectively induce apoptosis in tumor cells and trigger a specific immune response. Similar results in nude mice were obtained with a vaccinia oncolytic virus by Belin et al. (2013). Finally, the first clinical results were obtained by a replication restricted herpes virus, HSV1716, in a phase I/II trial (Danson 
et al., 2020). Thirteen patients with advanced MMe, 8 of which were treatment-naïve, received intrapleural injections of this virus (Figure 1). Despite the absence of objective responses, disease stabilization was achieved in $50 \%$ of patients and no major adverse events were reported. Moreover, active viral replication and Th1 cytokines were documented in the pleural fluid of the patients. This study opens up to the possibility of an oncolytic virus therapy in $\mathrm{MMe}$, however, studies of combination strategies, maybe with ICIs, could be beneficial.

\section{Hypoxia}

Like many other forms of cancer, MMe shows a metabolic rewiring finalized to maintain the production of energy and to sustain the anabolic pathways in a hostile microenvironment. This is possible through the activation of specific regulators such as the Hypoxia Inducible Factors $1 \alpha$ and $2 \alpha$ (HIF- $1 \alpha$ and HIF$2 \alpha$ ). Targeting this survival mechanism has longly been attempted with mixed results, in many tumors. An interesting success was recently obtained in Renal Cell Carcinoma associated with the Von Hippel-Lindau disease, by the HIF-2 $\alpha$ inhibitor Belzutifan, which reached an ORR of 49\% (Jonasch et al., 2021).

In MMe, this approach has been exploited only recently and in a preclinical setting. Shukuya et al., for example, demonstrated that MMe cells that carry a mutation of the metabolic regulator VHL (Von Hippel Lindau) can sensibly slow down their growth rate when exposed to the HIF-1 $\alpha$ inhibitor YC-1 (Shukuya et al., 2020). Similarly, Li Petri et al. showed that MMe cells tend to seek a hypoxic status in order to decrease the expression of protoncoupled folate transporter (PCFT), which is associated with improved survival of $\mathrm{MMe}$ patients treated with pemetrexed (Li Petri et al., 2020). In the same work, they demonstrated, both in vitro and in vivo, that the inhibition of the lactate dehydrogenase enzyme through the novel inhibitor NHI-Glc-2

\section{REFERENCES}

Adusumilli, P. S., Zauderer, M. G., and Rivière, I. (2021). "A Phase I Trial of Regional Mesothelin-Targeted CAR T-cell Therapy in Patients with Malignant Pleural Disease, in Combination with the Anti-PD-1 Agent Pembrolizumab. Cancer Discov. 11 (11), 2748-2763. doi:10.1158/2159-8290.CD-21-0407

Aliagas, E., Alay, A., and Martínez-Iniesta, M. (2021). Efficacy of CDK4/6 Inhibitors in Preclinical Models of Malignant Pleural Mesothelioma. $\mathrm{Br}$. J. Cancer 125 (10), 1365-1376. doi:10.1038/s41416-021-01547-y

Andreea, V., Capucine, B., and Patricia, M. (2018). Safety and Efficacy Results from a Phase I Dose-Escalation Trial of Nintedanib in Combination with Pembrolizumab in Patients with Advanced Solid Tumors (PEMBIB Trial). J. Clin. Oncol. 36 (15), 3080.

Andtbacka, R. H. I., Collichio, F., Harrington, K. J., Middleton, M. R., Downey, G., Öhrling, K., et al. (2019). Final Analyses of OPTiM: a Randomized Phase III Trial of Talimogene Laherparepvec versus Granulocyte-Macrophage colonystimulating Factor in Unresectable Stage III-IV Melanoma. J. Immunother. Cancer 7 (1), 145. doi:10.1186/s40425-019-0623-Z

Anobile, D. P., Bironzo, P., Picca, F., Lingua, M. F., Morena, D., Righi, L., et al. (2021). Evaluation of the Preclinical Efficacy of Lurbinectedin in Malignant Pleural Mesothelioma. Cancers (Basel) 13 (10), 2332. doi:10.3390/ cancers13102332

Aoe, K., Hiraki, A., Tanaka, T., Gemba, K., Taguchi, K., Murakami, T., et al. (2006). Expression of Vascular Endothelial Growth Factor in Malignant Mesothelioma. Anticancer Res. 26 (6C), 4833-4836. causes the disruption of the MMe spheroid cell cultures and exert a marked antitumor efficacy in the mice. The effect of this agent seems to be synergistic with some of the chemotherapy drugs already used in the clinical practice, such as Pemetrexed and Gemcitabine.

\section{CONCLUSION}

Until recently, MMe was an orphan disease, endowed by a dramatic prognosis which benefited little from available, mainly chemotherapy-based, treatments.

More recently, ICIs have represented an improvement in the palliative treatment setting of this disease; although, this improvement proved to be small, it represents a first step towards more active novel treatment options.

Unbiased trials, possibly conducted with novel designs (e.g., adaptive studies) are still needed, also in order to improve the cost:ratio benefit of the next generation of agents. Indeed, the small amount of benefit achieved so far should be weighted against the costs of novel anticancer agents and strategies.

The preclinical and early clinical studies here discussed offer a glimpse of what the therapy of MMe could look like in the next future, offering hopes for our patients. It is however clear, in our opinion, that the road towards more effective treatments for MMe ahead of us is still long and complex.

\section{AUTHOR CONTRIBUTIONS}

GP: first author, conceptualization and writing MR, MP, and SM: proof-reading CP and LM: co-senior authors, supervision and final editing.

Baas, P., Scherpereel, A., and Nowak, A. (2021). First-line Nivolumab Plus Ipilimumab in Unresectable Malignant Pleural Mesothelioma (CheckMate 743): a Multicentre, Randomised, Open-Label, Phase 3 Trial. Lancet 397 (10272), 670. doi:10.1016/s0140-6736(20)32714-8

Bearz, A., Talamini, R., Rossoni, G., Santo, A., de Pangher, V., Fasola, G., et al. (2012). Re-challenge with Pemetrexed in Advanced Mesothelioma: a MultiInstitutional Experience. BMC Res. Notes 5, 482. doi:10.1186/1756-0500-5-482

Beatty, G. L., Haas, A. R., Maus, M. V., Torigian, D. A., Soulen, M. C., Plesa, G., et al. (2015). Mesothelin-specific Chimeric Antigen Receptor mRNAEngineered T Cells Induce Anti-tumor Activity in Solid Malignancies. Cancer Immunol. Res. 2 (2), 112-120. doi:10.1158/2326-6066.CIR-130170

Belin, L. J., Ady, J. W., Lewis, C., Marano, D., Gholami, S., Mojica, K., et al. (2013). An Oncolytic Vaccinia Virus Expressing the Human Sodium Iodine Symporter Prolongs Survival and Facilitates SPECT/CT Imaging in an Orthotopic Model of Malignant Pleural Mesothelioma. Surgery 154 (3), 486-495. doi:10.1016/ j.surg.2013.06.004

Bensaid, D., Blondy, T., Deshayes, S., Dehame, V., Bertrand, P., Grégoire, M., et al. (2018). Assessment of New HDAC Inhibitors for Immunotherapy of Malignant Pleural Mesothelioma. Clin. Epigenetics 10, 79. doi:10.1186/s13148-018-0517-9

Bononi, A., Napolitano, A., Pass, H. I., Yang, H., and Carbone, M. (2015). Latest Developments in Our Understanding of the Pathogenesis of Mesothelioma and the Design of Targeted Therapies. Expert Rev. Respir. Med. 9 (5), 633-654. doi:10.1586/17476348.2015.1081066

Campbell, N. P., Kunnavakkam, R., Leighl, N., Vincent, M. D., Gandara, D. R., Koczywas, M., et al. (2012). Cediranib in Patients with Malignant 
Mesothelioma: a Phase II Trial of the University of Chicago Phase II Consortium. Lung Cancer 78 (1), 76-80. doi:10.1016/j.lungcan.2012.06.011

Ceresoli, G. L., Zucali, P. A., Favaretto, A. G., Grossi, F., Bidoli, P., Del Conte, G., et al. (2006). Phase II Study of Pemetrexed Plus Carboplatin in Malignant Pleural Mesothelioma. J. Clin. Oncol. 24 (9), 1443-1448. doi:10.1200/ JCO.2005.04.3190

Cho, B. C. J., Donahoe, L., Bradbury, P. A., Leighl, N., Keshavjee, S., Hope, A., et al. (2021). Surgery for Malignant Pleural Mesothelioma after Radiotherapy (SMART): Final Results from a single-centre, Phase 2 Trial. Lancet Oncol. 22 (2), 190-197. doi:10.1016/S1470-2045(20)30606-9

Crisanti, M. C., Wallace, A. F., Kapoor, V., Vandermeers, F., Dowling, M. L., Pereira, L. P., et al. (2009). The HDAC Inhibitor Panobinostat (LBH589) Inhibits Mesothelioma and Lung Cancer Cells In Vitro and In Vivo with Particular Efficacy for Small Cell Lung Cancer. Mol. Cancer Ther. 8 (8), 2221-2231. doi:10.1158/1535-7163.MCT-09-0138

Danson, S. J., Conner, J., Edwards, J. G., Blyth, K. G., Fisher, P. M., Muthana, M., et al. (2020). Oncolytic Herpesvirus Therapy for Mesothelioma - A Phase I/IIa Trial of Intrapleural Administration of HSV1716. Lung Cancer 150, 145-151. doi:10.1016/j.lungcan.2020.10.007

Dudek, A. Z., Wang, X., Gu, L., Duong, S., Stinchcombe, T. E., Kratzke, R., et al. (2020). Randomized Study of Maintenance Pemetrexed versus Observation for Treatment of Malignant Pleural Mesothelioma: CALGB 30901. Clin. Lung Cancer 21 (6), 553-e1. doi:10.1016/j.cllc.2020.06.025

Fennell, D. A., Kirkpatrick, E., Cozens, K., Nye, M., Lester, J., Hanna, G., et al. (2018). CONFIRM: a Double-Blind, Placebo-Controlled Phase III Clinical Trial Investigating the Effect of Nivolumab in Patients with Relapsed Mesothelioma: Study Protocol for a Randomised Controlled Trial. Trials 19 (1), 233. doi:10.1186/s13063-018-2602-y

Garland, L. L., Chansky, K., Wozniak, A. J., Tsao, A. S., Gadgeel, S. M., Verschraegen, C. F., et al. (2011). Phase II Study of Cediranib in Patients with Malignant Pleural Mesothelioma: SWOG S0509. J. Thorac. Oncol. 6 (11), 1938-1945. doi:10.1097/JTO.0b013e318229586e

Garland, L. L., Rankin, C., Gandara, D. R., Rivkin, S. E., Scott, K. M., Nagle, R. B., et al. (2007). Phase II Study of Erlotinib in Patients with Malignant Pleural Mesothelioma: a Southwest Oncology Group Study. J. Clin. Oncol. 25 (17), 2406-2413. doi:10.1200/JCO.2006.09.7634

Haas, A. R., Tanyi, J. L., O’Hara, M. H., Gladney, W. L., Lacey, S. F., Torigian, D. A., et al. (2019). Phase I Study of Lentiviral-Transduced Chimeric Antigen Receptor-Modified T Cells Recognizing Mesothelin in Advanced Solid Cancers. Mol. Ther. 27 (11), 1919-1929. doi:10.1016/j.ymthe.2019.07.015

Hassan, R., Alley, E., Kindler, H., Antonia, S., Jahan, T., Honarmand, S., et al. (2019). Clinical Response of Live-Attenuated, Listeria Monocytogenes Expressing Mesothelin (CRS-207) with Chemotherapy in Patients with Malignant Pleural Mesothelioma. Clin. Cancer Res. 25 (19), 5787-5798. doi:10.1158/1078-0432.CCR-19-0070

Hassan, R., Blumenschein, G. R., Moore, K. N., Santin, A. D., Kindler, H. L., Nemunaitis, J. J., et al. (2020). First-in-Human, Multicenter, Phase I DoseEscalation and Expansion Study of Anti-mesothelin Antibody-Drug Conjugate Anetumab Ravtansine in Advanced or Metastatic Solid Tumors. J. Clin. Oncol. 38 (18), 1824-1835. doi:10.1200/JCO.19.02085

Inglis, D. J., Lavranos, T. C., Beaumont, D. M., Leske, A. F., Brown, C. K., Hall, A. J., et al. (2014). The Vascular Disrupting Agent BNC105 Potentiates the Efficacy of VEGF and mTOR Inhibitors in Renal and Breast Cancer. Cancer Biol. Ther. 15 (11), 1552-1560. doi:10.4161/15384047.2014.956605

Ivanov, S. V., Miller, J., Lucito, R., Tang, C., Ivanova, A. V., Pei, J., et al. (2009). Genomic Events Associated with Progression of Pleural Malignant Mesothelioma. Int. J. Cancer 124 (3), 589-599. doi:10.1002/ijc.23949

Jang, H., Truong, C., and Lo, E. (2021). Inhibition of CDK4/6 Overcomes Primary Resistance to PD-1 Blockade in Malignant Mesothelioma. Ann. Thorac. Surg. S0003-4975 (21), 01670-01672. doi:10.1016/j.athoracsur.2021.08.054

Jonasch, E., Donskov, F., Iliopoulos, O., Rathmell, W. K., Narayan, V. K., Maughan, B. L., et al. (2021). Belzutifan for Renal Cell Carcinoma in von Hippel-Lindau Disease. N. Engl. J. Med. 385 (22), 2036-2046. doi:10.1056/ NEJMoa2103425

Kanteti, R., Mirzapoiazova, T., Riehm, J. J., Dhanasingh, I., Mambetsariev, B., Wang, J., et al. (2018). Focal Adhesion Kinase a Potential Therapeutic Target for Pancreatic Cancer and Malignant Pleural Mesothelioma. Cancer Biol. Ther. 19 (4), 316-327. doi:10.1080/15384047.2017.1416937
Keshava, H. B., Tang, A., Siddiqui, H. U., Raja, S., Raymond, D. P., Bribriesco, A., et al. (2019). Largely Unchanged Annual Incidence and Overall Survival of Pleural Mesothelioma in the USA. World J. Surg. 43 (12), 3239-3247. doi:10.1007/s00268-019-05132-6

Krug, L. M., Kindler, H. L., Calvert, H., Manegold, C., Tsao, A. S., Fennell, D., et al. (2015). Vorinostat in Patients with Advanced Malignant Pleural Mesothelioma Who Have Progressed on Previous Chemotherapy (VANTAGE-014): a Phase 3, Double-Blind, Randomised, Placebo-Controlled Trial. Lancet Oncol. 16 (4), 447-456. doi:10.1016/S1470-2045(15)70056-2

Kucharczuk, J. C., Randazzo, B., Chang, M. Y., Amin, K. M., Elshami, A. A., Sterman, D. H., et al. (1997). Use of a "Replication-Restricted" Herpes Virus to Treat Experimental Human Malignant Mesothelioma. Cancer Res. 57 (3), 466-471.

Lam, W. S., Creaney, J., Chen, F. K., Chin, W. L., Muruganandan, S., Arunachalam, S., et al. (2020). A Phase II Trial of Single Oral FGF Inhibitor, AZD4547, as Second or Third Line Therapy in Malignant Pleural Mesothelioma. Lung Cancer 140, 87-92. doi:10.1016/j.lungcan.2019.12.018

Le, D. T., Wang-Gillam, A., Picozzi, V., Greten, T. F., Crocenzi, T., Springett, G., et al. (2015). Safety and Survival with GVAX Pancreas Prime and Listeria Monocytogenes-Expressing Mesothelin (CRS-207) Boost Vaccines for Metastatic Pancreatic Cancer. J. Clin. Oncol. 33 (12), 1325-1333. doi:10.1200/JCO.2014.57.4244

Li, H., Peng, K. W., Dingli, D., Kratzke, R. A., and Russell, S. J. (2010). Oncolytic Measles Viruses Encoding Interferon Beta and the Thyroidal Sodium Iodide Symporter Gene for Mesothelioma Virotherapy. Cancer Gene Ther. 17 (8), 550-558. doi:10.1038/cgt.2010.10

Li Petri, G., El Hassouni, B., Sciarrillo, R., Funel, N., Mantini, G., Zeeuw van der Laan, E. A., et al. (2020). Impact of Hypoxia on Chemoresistance of Mesothelioma Mediated by the Proton-Coupled Folate Transporter, and Preclinical Activity of New Anti-LDH-A Compounds. Br. J. Cancer 123 (4), 644-656. doi:10.1038/s41416-020-0912-9

Marjorie, G., Peter, W., and Sylvestre, L. (2020). Safety and Efficacy of Tazemetostat, an Enhancer of Zeste-Homolog 2 Inhibitor, in Patients with Relapsed or Refractory Malignant Mesothelioma. J. Clin. Oncol. 38 (15), 9058.

Metaxas, Y., Früh, M., Eboulet, E. I., Grosso, F., Pless, M., Zucali, P. A., et al. (2020). Lurbinectedin as Second- or Third-Line Palliative Therapy in Malignant Pleural Mesothelioma: an International, multi-centre, Single-Arm, Phase II Trial (SAKK 17/16). Ann. Oncol. 31 (4), 495-500. doi:10.1016/j.annonc.2019.12.009

Nicholson, A. G., Sauter, J. L., Nowak, A. K., Kindler, H. L., Gill, R. R., Remy-Jardin, M., et al. (2020). EURACAN/IASLC Proposals for Updating the Histologic Classification of Pleural Mesothelioma: Towards a More Multidisciplinary Approach. J. Thorac. Oncol. 15 (1), 29-49. doi:10.1016/j.jtho.2019.08.2506

Nowak, A. K., Brown, C., Millward, M. J., Creaney, J., Byrne, M. J., Hughes, B., et al. (2013). A Phase II Clinical Trial of the Vascular Disrupting Agent BNC105P as Second Line Chemotherapy for Advanced Malignant Pleural Mesothelioma. Lung Cancer 81 (3), 422-427. doi:10.1016/j.lungcan.2013.05.006

Nowak, A. K., Lesterhuis, W. J., Kok, P. S., Brown, C., Hughes, B. G., Karikios, D. J., et al. (2020). Durvalumab with First-Line Chemotherapy in Previously Untreated Malignant Pleural Mesothelioma (DREAM): a Multicentre, Single-Arm, Phase 2 Trial with a Safety Run-In. Lancet Oncol. 21 (9), 1213-1223. doi:10.1016/S1470-2045(20)30462-9

Odgerel, C. O., Takahashi, K., Sorahan, T., Driscoll, T., Fitzmaurice, C., Yoko-O, M., et al. (2017). Estimation of the Global burden of Mesothelioma Deaths from Incomplete National Mortality Data. Occup. Environ. Med. 74 (12), 851-858. doi:10.1136/oemed-2017-104298

Peng, Y., and Croce, C. M. (2016). The Role of MicroRNAs in Human Cancer. Signal. Transduct Target. Ther. 1, 15004. doi:10.1038/sigtrans.2015.4

Pinto, C., Zucali, P. A., Pagano, M., Grosso, F., Pasello, G., Garassino, M. C., et al. (2021). Gemcitabine with or without Ramucirumab as Second-Line Treatment for Malignant Pleural Mesothelioma (RAMES): a Randomised, Double-Blind, Placebo-Controlled, Phase 2 Trial. Lancet Oncol. 22 (10), 1438-1447. doi:10.1016/S1470-2045(21)00404-6

Popat, S., Curioni-Fontecedro, A., Dafni, U., Shah, R., O’Brien, M., Pope, A., et al. (2020). A Multicentre Randomised Phase III Trial Comparing Pembrolizumab versus Single-Agent Chemotherapy for Advanced Pre-treated Malignant Pleural Mesothelioma: the European Thoracic Oncology Platform (ETOP 915) PROMISE-Meso Trial. Ann. Oncol. 31 (12), 1734-1745. doi:10.1016/ j.annonc.2020.09.009 
Porta, C., Nardone, V., Gray, S., Correale, P., and Mutti, L. (2021). RAMES Study: Is There Really a Role of VEGF-Inhibition in Mesothelioma? Lancet Oncol. 22 (12), e533. doi:10.1016/S1470-2045(21)00666-5

Raghav, K., Liu, S., and Overman, M. J. (2021). Efficacy, Safety, and Biomarker Analysis of Combined PD-L1 (Atezolizumab) and VEGF (Bevacizumab) Blockade in Advanced Malignant Peritoneal Mesothelioma. Cancer Discov. 11 (11), 2738-2747. doi:10.1158/2159-8290.CD-21-0331

Rathkey, D., Khanal, M., Murai, J., Zhang, J., Sengupta, M., Jiang, Q., et al. (2020). Sensitivity of Mesothelioma Cells to PARP Inhibitors Is Not Dependent on BAP1 but Is Enhanced by Temozolomide in Cells with High-Schlafen 11 and Low-O6-Methylguanine-DNA Methyltransferase Expression. J. Thorac. Oncol. 15 (5), 843-859. doi:10.1016/j.jtho.2020.01.012

Reid, G., Pel, M. E., Kirschner, M. B., Cheng, Y. Y., Mugridge, N., Weiss, J., et al. (2013). Restoring Expression of miR-16: a Novel Approach to Therapy for Malignant Pleural Mesothelioma. Ann. Oncol. 24 (12), 3128-3135. doi:10.1093/ annonc/mdt 412

Ricciardi, S., Cardillo, G., Zirafa, C. C., Carleo, F., Facciolo, F., Fontanini, G., et al. (2018). Surgery for Malignant Pleural Mesothelioma: an International Guidelines Review. J. Thorac. Dis. 10 (Suppl. 2), S285-S292. doi:10.21037/ jtd.2017.10.16

Rini, B. I., Plimack, E. R., Stus, V., Gafanov, R., Hawkins, R., Nosov, D., et al. (2019). Pembrolizumab Plus Axitinib versus Sunitinib for Advanced Renal-Cell Carcinoma. N. Engl. J. Med. 380 (12), 1116-1127. doi:10.1056/NEJMoa1816714

Santamaría Nuñez, G., Robles, C., and Giraudon, C. (2016). Lurbinectedin Specifically Triggers the Degradation of Phosphorylated RNA Polymerase II and the Formation of DNA Breaks in Cancer Cells. Mol. Cancer Ther. 15 (10), 2399-2412.

Seiji, M., Akihiro, F., and Toru, N. (2018). CDK4/6 Inhibitor and Radiation Therapy in Malignant Pleural Mesothelioma. J. Clin. Oncol. 36 (15), e24326-e24326. doi:10.1200/JCO.2018.36.15

Shukuya, T., Oyanagi, J., Serizawa, M., Watanabe, M., Yamamoto, N., and Koh, Y. (2020). Hypoxia Inducible Factor-1a Inhibition in Von Hippel Lindau-Mutant Malignant Pleural Mesothelioma Cells. Anticancer Res. 40 (4), 1867-1874. doi:10.21873/anticanres.14140

Singh, P. K., Deorukhkar, A. A., Venkatesulu, B. P., Li, X., Tailor, R., Bomalaski, J. S., et al. (2019). Exploiting Arginine Auxotrophy with Pegylated Arginine Deiminase (ADI-PEG20) to Sensitize Pancreatic Cancer to Radiotherapy via Metabolic Dysregulation. Mol. Cancer Ther. 18 (12), 2381-2393. doi:10.1158/ 1535-7163.MCT-18-0708

Soria, J. C., Gan, H. K., Blagden, S. P., Plummer, R., Arkenau, H. T., Ranson, M., et al. (2016). A Phase I, Pharmacokinetic and Pharmacodynamic Study of GSK2256098, a Focal Adhesion Kinase Inhibitor, in Patients with Advanced Solid Tumors. Ann. Oncol. 27 (12), 2268-2274. doi:10.1093/annonc/mdw427

Spring, L. M., Wander, S. A., Zangardi, M., and Bardia, A. (2019). CDK 4/6 Inhibitors in Breast Cancer: Current Controversies and Future Directions. Curr. Oncol. Rep. 21 (3), 25. doi:10.1007/s11912-019-0769-3

Tomasetti, M., Amati, M., Nocchi, L., Saccucci, F., Strafella, E., Staffolani, S., et al. (2011). Asbestos Exposure Affects poly(ADP-Ribose) Polymerase-1 Activity: Role in Asbestos-Induced Carcinogenesis. Mutagenesis 26 (5), 585-591. doi:10.1093/mutage/ger020

Treasure, T., Lang-Lazdunski, L., Waller, D., Bliss, J. M., Tan, C., Entwisle, J., et al. (2011). Extra-pleural Pneumonectomy versus No Extra-pleural Pneumonectomy for Patients with Malignant Pleural Mesothelioma: Clinical Outcomes of the Mesothelioma and Radical Surgery (MARS) Randomised Feasibility Study. Lancet Oncol. 12 (8), 763-772. doi:10.1016/S1470-2045(11) 70149-8

Trigo, J., Subbiah, V., Besse, B., Moreno, V., López, R., Sala, M. A., et al. (2020). Lurbinectedin as Second-Line Treatment for Patients with Small-Cell Lung Cancer: a Single-Arm, Open-Label, Phase 2 Basket Trial. Lancet Oncol. 21 (5), 645-654. doi:10.1016/S1470-2045(20)30068-1
Tsao, A. S., Miao, J., Wistuba, I. I., Vogelzang, N. J., Heymach, J. V., Fossella, F. V., et al. (2019). Phase II Trial of Cediranib in Combination with Cisplatin and Pemetrexed in Chemotherapy-Naïve Patients with Unresectable Malignant Pleural Mesothelioma (SWOG S0905). J. Clin. Oncol. 37 (28), 2537-2547. doi:10.1200/JCO.19.00269

van Zandwijk, N., Pavlakis, N., Kao, S. C., Linton, A., Boyer, M. J., Clarke, S., et al. (2017). Safety and Activity of microRNA-Loaded Minicells in Patients with Recurrent Malignant Pleural Mesothelioma: a First-In-Man, Phase 1, OpenLabel, Dose-Escalation Study. Lancet Oncol. 18 (10), 1386-1396. doi:10.1016/ S1470-2045(17)30621-6

Vlacic, G., Hoda, M. A., Klikovits, T., Sinn, K., Gschwandtner, E., Mohorcic, K., et al. (2019). Expression of FGFR1-4 in Malignant Pleural Mesothelioma Tissue and Corresponding Cell Lines and its Relationship to Patient Survival and FGFR Inhibitor Sensitivity. Cells 8 (9), 1091. doi:10.3390/cells8091091

Vogelzang, N. J., Rusthoven, J. J., Symanowski, J., Denham, C., Kaukel, E., Ruffie, P., et al. (2003). Phase III Study of Pemetrexed in Combination with Cisplatin versus Cisplatin Alone in Patients with Malignant Pleural Mesothelioma. J. Clin. Oncol. 21 (14), 2636-2644. doi:10.1200/JCO.2003.11.136

Voigt, S. L., Raman, V., Jawitz, O. K., Bishawi, M., Yang, C. J., Tong, B. C., et al. (2020). The Role of Neoadjuvant Chemotherapy in Patients with Resectable Malignant Pleural Mesothelioma-An Institutional and National Analysis. J. Natl. Cancer Inst. 112 (11), 1118-1127. doi:10.1093/jnci/djaa002

Zalcman, G., Mazieres, J., Margery, J., Greillier, L., Audigier-Valette, C., MoroSibilot, D., et al. (2016). Bevacizumab for Newly Diagnosed Pleural Mesothelioma in the Mesothelioma Avastin Cisplatin Pemetrexed Study (MAPS): a Randomised, Controlled, Open-Label, Phase 3 Trial. Lancet 387 (10026), 1405-1414. doi:10.1016/S0140-6736(15)01238-6

Zucali, P. A., Perrino, M., Lorenzi, E., Ceresoli, G. L., De Vincenzo, F., Simonelli, M., et al. (2014). Vinorelbine in Pemetrexed-Pretreated Patients with Malignant Pleural Mesothelioma. Lung Cancer 84 (3), 265-270. doi:10.1016/ j.lungcan.2013.11.011

Conflict of Interest: MR reports personal fees from MSD, personal fees from Pfizer, personal fees from Novartis, personal fees from AstraZeneca, during the conduct of the study. CP reports personal fees from Angelini Pharma, personal fees from AstraZeneca, personal fees from BMS, personal fees from Eisai, personal fees from EUSA Pharma, personal fees from General Electric, personal fees from Ipsen, personal fees from Janssen, personal fees from Merck Serono, personal fees from MSD, personal fees from Novartis, personal fees from Pfizer, and Roche, outside the submitted work.

The remaining authors declare that the research was conducted in the absence of any commercial or financial relationships that could be construed as a potential conflict of interest.

Publisher's Note: All claims expressed in this article are solely those of the authors and do not necessarily represent those of their affiliated organizations, or those of the publisher, the editors and the reviewers. Any product that may be evaluated in this article, or claim that may be made by its manufacturer, is not guaranteed or endorsed by the publisher.

Copyright $\odot 2021$ Pezzicoli, Rizzo, Perrone, Minei, Mutti and Porta. This is an openaccess article distributed under the terms of the Creative Commons Attribution License (CC BY). The use, distribution or reproduction in other forums is permitted, provided the original author(s) and the copyright owner(s) are credited and that the original publication in this journal is cited, in accordance with accepted academic practice. No use, distribution or reproduction is permitted which does not comply with these terms. 